\title{
Alignment of Carbon Nanotubes under low magnetic fields through attachment of magnetic nanoparticles
}

Miguel A. Correa-Duarte ${ }^{1,2} *$, Marek Grzelczak, ${ }^{1}$ Verónica Salgueiriño-Maceira, ${ }^{3}$ Michael Giersig, ${ }^{4}$ Luis M. Liz-Marzán, ${ }^{1}$ Michael Farle, ${ }^{5}$ Karl Sierazdki, ${ }^{2}$ Rodolfo Diaz ${ }^{6}$

${ }^{1}$ Departamento de Química-Física, Universidade de Vigo, E-36310, Vigo (Spain)

${ }^{2}$ Department of Mechanical and Aerospace Engineering, Arizona State University, Tempe 85281 (USA)

${ }^{3}$ Instituto de Investigacións Tecnolóxicas, Universidade de Santiago de Compostela, E-15782, Santiago de Compostela (Spain)

${ }^{4}$ Center of Advanced European Studies and Research (CAESAR), Ludwig-Erhard-Allee 2, 53175 Bonn

(Germany)

${ }^{5}$ Fachbereich Physik, Universtität Duisburg-Essen, D-47048 Duisburg (Germany)

${ }^{6}$ Department of Electrical Engineering, Arizona State University, Tempe 85281 (USA)

e-mail: macorrea@uvigo.es

\section{SUPPORTING INFORMATION}

\section{Experimental}

$\mathrm{FeCl}_{3}, \mathrm{FeSO}_{4}$, tetramethylammonium hydroxide, poly-(diallyldimethylammonium chloride) (PDDA), $\mathrm{M}_{\mathrm{w}}<500,000$, and poly(sodium 4-styrenesulfonate) (PSS), $\mathrm{M}_{\mathrm{w}}$ 70000, were purchased from Aldrich. Sodium chloride was obtained from Merck. Hydrochloric Acid was purchased from Fluka. Milli-Q water with a resistivity higher than 18.2 $\mathrm{M} \Omega \mathrm{cm}$ was used in all experiments. MWNTs synthesized using plasma enhanced chemical vapor deposition (PECVD) ${ }^{[1]}$ were supplied by Nanolab (Boston) as a powder.

$\mathrm{Fe}_{3} \mathrm{O}_{4} / \gamma-\mathrm{Fe}_{2} \mathrm{O}_{3}$ nanoparticles. Aqueous dispersions of magnetite nanoparticles which were partially oxidized to maghemite were prepared according to Massart's method, ${ }^{2}$ based on the co-precipitation of ferrous and ferric ion solutions (1:2 molar ratio). $20 \mathrm{~mL}$ of aqueous $1 \mathrm{M} \mathrm{FeCl}_{3}$ and $5 \mathrm{~mL}$ of $2 \mathrm{M} \mathrm{FeSO}_{4}$ in $2 \mathrm{M} \mathrm{HCl}$ were added to $250 \mathrm{~mL}$ of $\mathrm{NH}_{4} \mathrm{OH}$ 0.7M under rapid mechanical stirring. Stirring was allowed to continue for 30 min, and then the black solid product was allowed to precipitate. The sediment was 
redispersed in $50 \mathrm{~mL}$ of distilled water, and subsequently three aliquots of $10 \mathrm{~mL}$ tetramethylammonium hydroxide solution (1M) were added, again with rapid stirring. Finally, water was added to the dispersion up to a total volume of $250 \mathrm{~mL}$. The particles have a diameter about 6-10 $\mathrm{nm}$.

$\mathrm{Fe}_{3} \mathrm{O}_{4} / \gamma-\mathrm{Fe}_{2} \mathrm{O}_{3}$-coated CNT. Polymer wrapping was performed following a method previously reported. Briefly, carbon nanotubes were dispersed in a $1 \mathrm{wt} \%$ aqueous solution of an anionic polyelectrolyte, PSS (polystyrene sulphonate) to a concentration of $150 \mathrm{mg} / \mathrm{L}$ by combination of strong stirring and sonication to ensure that well dispersed individual MWNTs are present in the dispersion, and the solution was stored for $12 \mathrm{~h}$ at $50^{\circ} \mathrm{C}$. Excess PSS was removed by three centrifugation/redispersion cycles, spinning at $12000 \mathrm{rpm}$ for $1 \mathrm{~h}$ and redispersing in pure water by stirring and brief sonication. Using this procedure, a stable colloid of PSS wrapped MWNTs in water was obtained.

After polymer wrapping with the anionic polyelectrolyte, the nanotubes were mixed with a $1 \mathrm{wt} \%$ aqueous solution of the cationic polyelectrolyte PDDA and $\mathrm{NaCl}(0.5 \mathrm{M})$ for 30 min. After three washing cycles by centrifugation at $12000 \mathrm{rpm}$ for $1 \mathrm{~h}$, the nanotubes were redispersed in $0.2 \mathrm{M} \mathrm{NaCl}$ and mixed with the $\mathrm{Fe}_{3} \mathrm{O}_{4} / \gamma-\mathrm{Fe}_{2} \mathrm{O}_{3}$ hydrosol during $1 \mathrm{~h}$. The obtained iron oxide nanoparticles-coated CNT where separated from the excess of nanoparticles by three washing-centrifugation cycles and finally redispersed in water at desired concentration.

\section{Magnetic measurements:}

In order to investigate the magnetic properties of $\mathrm{Fe}_{3} \mathrm{O}_{4} / \gamma-\mathrm{Fe}_{2} \mathrm{O}_{3}$ nanoparticles and $\mathrm{Fe}_{3} \mathrm{O}_{4} / \gamma-\mathrm{Fe}_{2} \mathrm{O}_{3}$-coated CNT they were mechanically compacted. The saturation magnetization measurements of $\mathrm{Fe}_{3} \mathrm{O}_{4} / \gamma-\mathrm{Fe}_{2} \mathrm{O}_{3}$ nanoparticles and $\mathrm{Fe}_{3} \mathrm{O}_{4} / \gamma-\mathrm{Fe}_{2} \mathrm{O}_{3}$-coated CNT are per gram of magnetic material (including the TMAOH as stabilizer in the first case and CNTs and polyelectrolytes in the second case). 


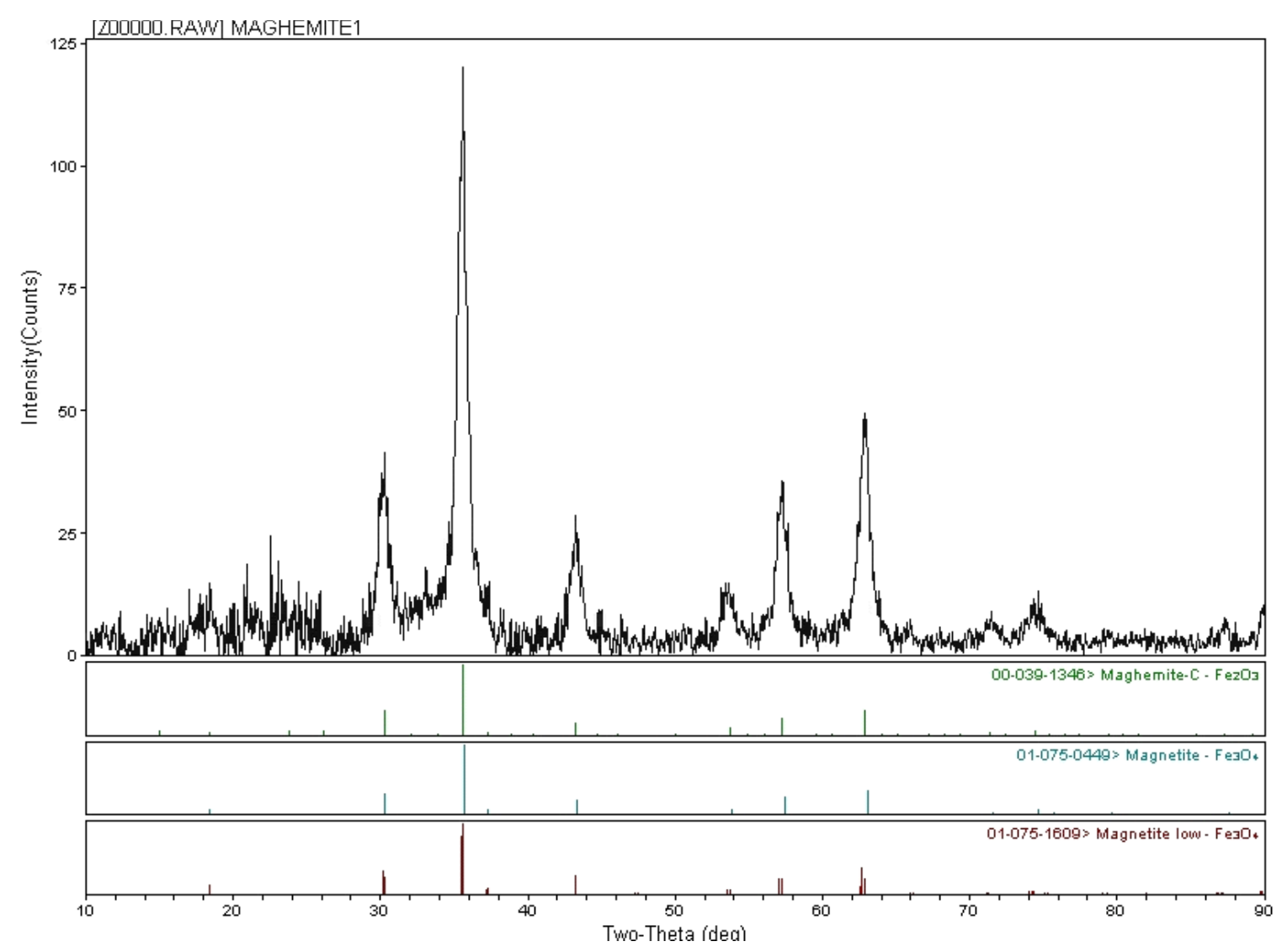

X-ray diffraction patterns for the $\mathrm{Fe}_{3} \mathrm{O}_{4} / \gamma \mathrm{Fe}_{2} \mathrm{O}_{3}$ nanoparticles used for the deposition.

${ }^{1}$ Z. F. Ren, Z. P. Huang, J. W. Xu, J. H. Wang, P. Bush, M. P. Siegal, P. N. Provencio, Science 1998, 282, 1105.

${ }^{2}$ Massart, R. IEEE Trans. Magn., 1981, MAG-17, 1247 